\title{
Pocket-creation method facilitates endoscopic submucosal dissection of colorectal laterally spreading tumors, non-granular type $\square$
}

\section{(9)( $\circledast \odot$}

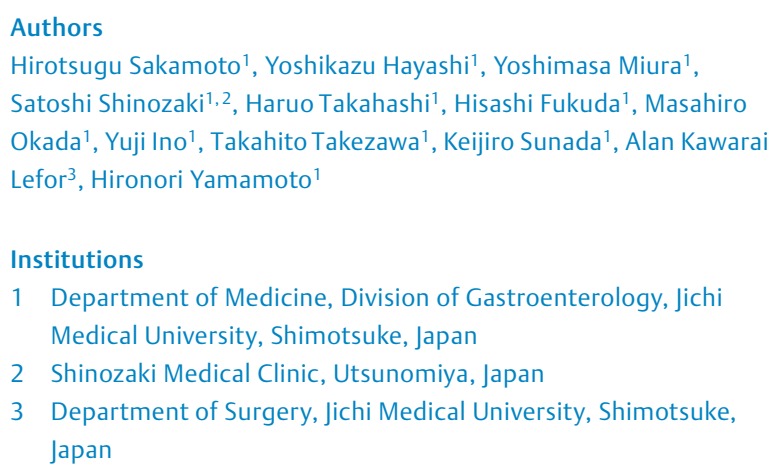

Hirotsugu Sakamoto ${ }^{1}$, Yoshikazu Hayashi ${ }^{1}$, Yoshimasa Miura ${ }^{1}$, Satoshi Shinozaki ${ }^{1,2}$, Haruo Takahashi', Hisashi Fukuda ${ }^{1}$, Masahiro Okada $^{1}$, Yuji Ino ${ }^{1}$, Takahito Takezawa ${ }^{1}$, Keijiro Sunada ${ }^{1}$, Alan Kawarai Lefor $^{3}$, Hironori Yamamoto ${ }^{1}$

Institutions

1 Department of Medicine, Division of Gastroenterology, Jichi Medical University, Shimotsuke, Japan

2 Shinozaki Medical Clinic, Utsunomiya, Japan

3 Department of Surgery, Jichi Medical University, Shimotsuke, Japan

submitted 5.8.2016

accepted after revision $\quad 16.11 .2016$

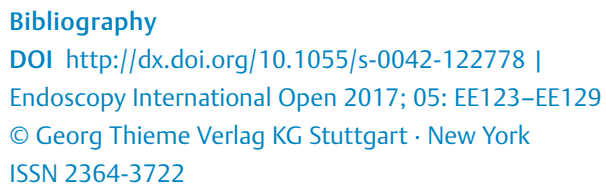

\begin{abstract}
Background and study aims The pocket-creation method (PCM) is a novel strategy for endoscopic submucosal dissection (ESD). The aim of this study is to determine the efficacy of the PCM for colorectal laterally spreading tumors, non-granular type (LST-NG).

Patients and methods The records of 126 consecutive patients with colorectal LST-NG who underwent ESD between April 2012 and July 2015 were retrospectively reviewed. Patients were divided into PCM $(n=73)$ and conventional method $(C M)(n=53)$ groups. Results The en bloc resection rate in the PCM group was significantly higher than in the CM group ( $100 \%$ [73/73] vs. $92 \%$ [49/53], $P=0.03)$. The en bloc resection rate with severe fibrosis was higher in the PCM group than in the CM group ( $100 \%$ [3/3] vs. $60 \%$ [3/5]). The R0 resection rate for the two groups was not statistically significantly different ( $93 \%$ [68/73] vs. $91 \%$ [48/53], $P=0.74$ ). The perforation rate in the PCM group was lower than in the CM group although not statistically significantly less $(0 \% 0 / 73$ vs. $4 \% 2 / 53, P=0.18)$. For lesions resected en bloc, dissection speed for the PCM group was significantly faster than for the CM group (median [IQR], 19 [13-24] vs. $\left.14[10-22] \mathrm{mm}^{2} / \mathrm{min}, P=0.03\right)$.

Conclusion ESD using PCM achieves a reliable and safe resection of colorectal LST-NG.
\end{abstract}

\section{Introduction}

Colorectal cancer is the third most commonly diagnosed cancer worldwide in males and the second in females as of 2012 [1]. Endoscopic mucosal resections (EMR) and conventional polypectomy are performed to remove premalignant or superficial lesions to reduce the incidence and mortality of colorectal cancer [2]. However, it is difficult to resect larger lesions (>20 mm) en bloc [3]. Endoscopic submucosal dissection (ESD) for colorectal neoplasms has become common in recent years. More than 18000 colorectal ESD procedures were performed in Japan between April 2014 and March 2015 [4]. ESD provides a higher en bloc resection rate with a lower recurrence rate compared with EMR [5]. However, colorectal ESD is considered more difficult than gastric ESD because the colorectal wall is thinner than in the stomach. Maneuverability of the endoscope in the colon is also poor due to the presence of semilunar folds, physiological flexion, peristalsis and respiratory movements.
Although various techniques and devices have been developed in order to overcome these difficulties, colorectal ESD has not become the standard approach except in East Asia [6,7]. Furthermore, standardization of the actual ESD procedure has not been achieved yet. A simple and safe ESD technique is needed to facilitate worldwide dissemination of the procedure for colorectal ESD.

Laterally spreading tumors, non-granular type (LST-NG), have a high frequency of submucosal invasion and fibrosis [8]. Therefore, en bloc resection with ESD should be used for LSTNG [9]. ESD for LST-NG is recommended to be performed only by endoscopists with advanced skills and extensive experience with colorectal ESD $[10,11]$.

The pocket-creation method (PCM) is a new strategy for ESD of superficial colorectal tumors $[12,13]$. The key feature of the PCM is creation of a large submucosal pocket using a small-caliber-tip transparent hood and needle-type knife. The PCM maintains a thick submucosal layer during the ESD procedure 
477 colorectal ESD (from April 2012 to July 2015) (460 patients)

124 non-LST lesions

(118 patients)

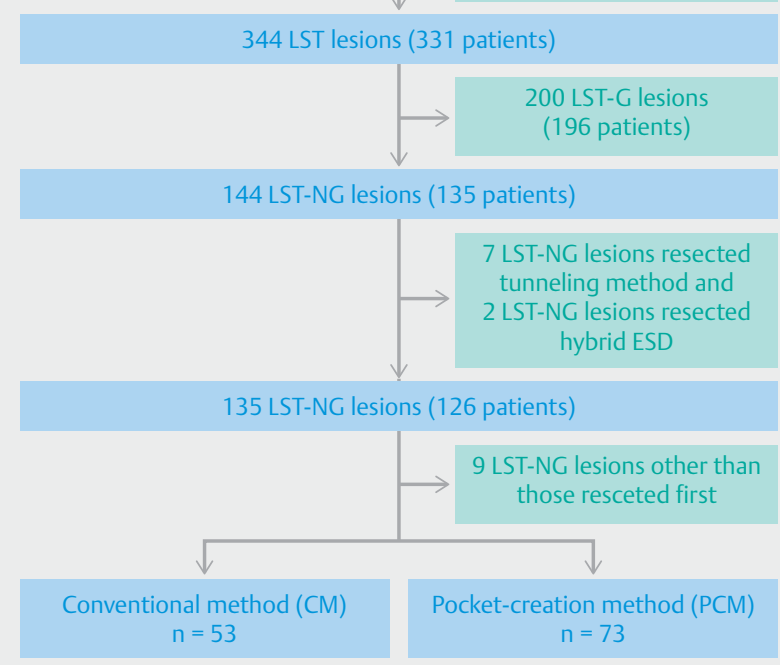

- Fig. 1 Flow chart of the study. because a minimal incision prevents dispersion of injected fluid. The tip of the endoscope in the submucosal pocket facilitates tissue traction, resulting in keeping the direction parallel to the muscularis [6]. However, there are no reports comparing the safety and efficacy of the PCM with conventional methods of resection. The aim of this study is to evaluate the usefulness of the PCM for colorectal LST-NG compared with the conventional method (CM).

\section{Patients and methods}

A total of 477 colorectal tumors in 460 patients were resected using ESD between April 2012 and July 2015 at Jichi Medical University Hospital. LST is defined as a flat neoplastic lesion larger than $10 \mathrm{~mm}$. LST is divided into two types based on endoscopic findings. The first type, laterally spreading tumors granular type (LST-G), has nodules or granules on the lesion, while LST-NG has a smooth surface without nodules or granules. Furthermore, LST-NG is divided into two subtypes, the pseudo-depressed type (LST-NG-PD) and the flat-elevated type (LST-NGF). The medical records of 331 consecutive patients with 344 colorectal LST were retrospectively reviewed. Two hundred LST-G lesions in 196 patients were excluded. Patients with recurrence after EMR were included in this study. Seven LST-NG lesions resected using the tunneling method and two LST-NG lesions resected using the hybrid ESD technique were excluded. Five patients had two LST-NG lesions resected and two patients had three LST-NG lesions. In patients with multiple lesions, only the first resected lesion is included in this study. Finally, a total of 126 LST-NG lesions in 126 patients were enrolled in this study ( $\triangleright$ Fig. 1). Of these, 73 lesions were removed using the PCM and 53 lesions were removed using the CM. Written in- formed consent for ESD of the colorectal lesions was obtained from all patients. This study was approved by the Institutional Review Board of Jichi Medical University Hospital.

\section{Method of colorectal ESD}

All patients were admitted before undergoing ESD. Bowel preparation was performed using $10 \mathrm{~mL}$ of oral $0.75 \%$ sodium picosulfate on the night before the procedure and $2 \mathrm{~L}$ of polyethylene glycol electrolyte solution on the day of the ESD procedure. When the stool became watery and clear, bowel preparation was considered complete even if $2 \mathrm{~L}$ of polyethylene glycol solution had not been taken. If the stools were not watery and clear after this, a further 1 to $2 \mathrm{~L}$ of polyethylene glycol electrolyte solution or 0.9 to $1.8 \mathrm{~L}$ of isotonic magnesium citrate solution were administered [14]. Midazolam and pethidine were used for sedation in most patients, and timepidium bromide hydrate or glucagon was used to decrease colonic peristalsis. All ESD procedures were performed using the CM from April 2012 to June 2013 during the study period. PCM was first performed in July 2013. After that, the method used to perform the ESD was decided by the physician in charge. If the ESD was performed by a trainee, at least 20 lesions had to have been resected with direct supervision by expert endoscopists.

\section{Endoscopic system and devices}

An endoscope with a waterjet instrument (EC-580RD/M; Fujifilm, Tokyo, Japan), carbon dioxide insufflation, and a small-caliber tip transparent (ST) hood (DH-15GR or DH-28GR; Fujifilm) fitted to the tip of the endoscope were used. When adequate maneuverability could not be maintained using a standard colonoscope, we used a balloon-assisted endoscope (EC-450BI5 and TS-13101; Fujifilm). For submucosal injection, $0.4 \%$ sodium hyaluronate solution (MucoUp; Seikagaku, Tokyo, Japan) with $0.002 \%-0.004 \%$ indigo carmine and $0.001 \%$ epinephrine was used. The mucosal incision and submucosal dissection were performed by using a Flush knife BT (DK2618JB-15; Fujifilm) or a DualKnife (KD-650Q; Olympus, Tokyo, Japan). Hot hemostatic forceps (HOYA Corporation, Tokyo, Japan) were used to control bleeding. A VIO300D (ERBE Elektromedizin $\mathrm{GmbH}$, Tübingen, Germany) electrosurgical generator was used. The mucosal incision was made with Endo-Cut I (effect, 1; duration, 4; interval, 1). Submucosal dissection was done using swift coagulation (effect 4, 25 W). Hemostasis was done with soft coagulation (effect 4, 80 W) [14].

\section{Conventional method (CM) [15] ( Fig. 2)}

First, the sodium hyaluronate solution with indigo carmine and epinephrine was injected into the submucosal layer under the lesion and the surrounding normal mucosa, which induced thickening of the submucosal layer and elevation of the lesion. An initial mucosal incision was made for at least one-quarter of the circumference approximately $5 \mathrm{~mm}$ from the distal side of the tumor. Then, submucosal dissection was performed from the distal to the proximal edge of the tumor. After dissecting all initial mucosal incision areas, additional mucosal incisions and submucosal dissection were repeated toward the proximal side. 


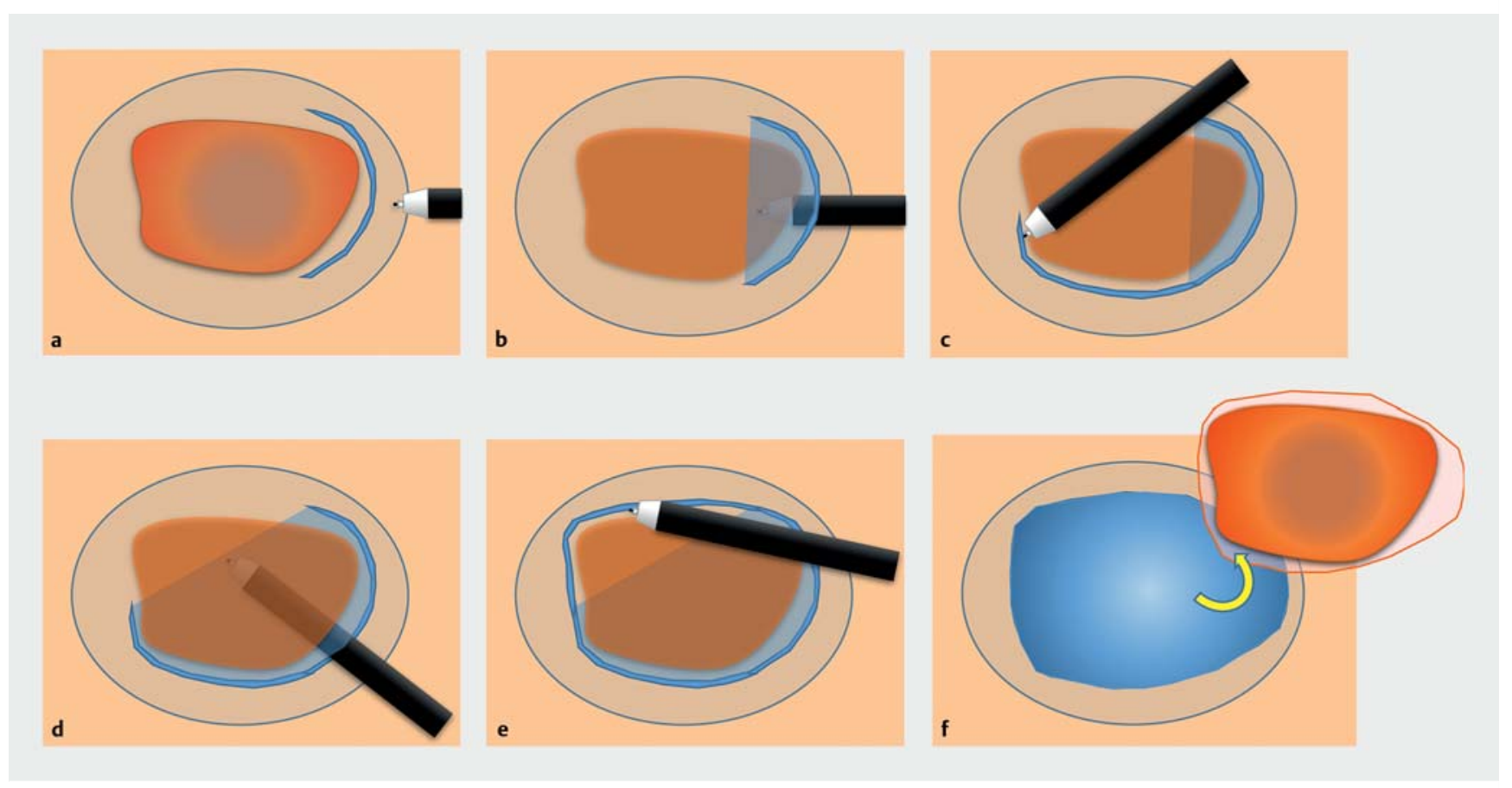

- Fig. 2 Sequence for the conventional method (CM) of colorectal endoscopic submucosal dissection [15]. First, $0.4 \%$ sodium hyaluronate solution is injected into the submucosal layer under the lesion and the surrounding normal mucosa. a An initial mucosal incision is made for at least one-quarter of the circumference and approximately $5 \mathrm{~mm}$ from the distal side. b Submucosal dissection is performed by sliding the knife from the center toward the side. $\mathbf{c}$ Making an additional mucosal incision in a step-by-step manner toward the proximal side. $\mathbf{d}$ Dissection of the incised area in the same manner. $\mathbf{e}$ Incision and dissection of the remaining area. $\mathbf{f}$ Completion of the en bloc resection.

\section{Pocket-creation method (PCM) [13] ( Fig. 3, ^Video 1)}

Submucosal injection was performed in the same manner as for the CM (above). An initial mucosal incision was made approximately $20 \mathrm{~mm}$ in length approximately $10 \mathrm{~mm}$ from the distal side of the tumor. Submucosal dissection was performed to make a pocket in the submucosal layer by inserting the tip of the endoscope with the ST hood under the mucosal tumor. The major difference from the CM is completion of the submucosal dissection under the lesion with a minimal mucosal incision in the PCM. After creation of a submucosal pocket under the tumor, an additional mucosal incision and submucosal dissection were made to open the lower side (based on the direction of gravity) of the pocket in a step-by-step manner toward the proximal side. Finally, a mucosal incision and submucosal dissection of the upper side were made in the same manner.

\section{Evaluation of colorectal ESD}

During the procedures, the presence and extent of submucosal fibrosis were assessed according to a previously reported system [16-18]. The classifications include: F0, no fibrosis, seen as a blue transparent submucosal layer; F1, mild fibrosis, which manifests as a white web-like structure in the blue submucosal layer; and F2, severe fibrosis, which is observed as a white structure without a blue transparent layer between the mucosal layer and the muscularis. Since the appearance of a white struc- ture is similar to the muscularis, it is difficult to distinguish from the muscularis.

After resection, we evaluated the size, shape, location, dissection time, and pathological findings for each lesion. The area of the resected specimen $\left(\mathrm{mm}^{2}\right)$ was calculated using the following formula:

Area $=$ Major axis $(\mathrm{mm}) / 2 \times$ Minor axis $(\mathrm{mm}) / 2 \times 3.14$ [14]

The dissection time (minutes) was defined from the start of cutting the mucosa to completion of the resection. The dissection speed $\left(\mathrm{mm}^{2} / \mathrm{min}\right)$ was calculated by using the area of the resected specimen $\left(\mathrm{mm}^{2}\right) /$ dissection time (minutes) [14]. En bloc resection was defined as a tumor removed as a single piece. $\mathrm{R} 0$ resection was defined as en bloc resection with pathologically negative resection margins. The definition of perforation included perforations both during and after the ESD procedure. Postoperative bleeding was defined as overt bleeding within 14 days after ESD requiring endoscopic hemostasis [14].

\section{Statistical analysis}

Statistical analysis was performed using EZR (version 1.32, Saitama Medical Center, Jichi Medical University, Saitama, Japan) [19]. Continuous variables were analyzed with the Student's $t$ test or the Mann-Whitney $U$ test and categorical data were compared using the Fisher's exact test. The impact of potential confounding factors was evaluated by using logistic regression analysis. All clinically important variables were included in the 

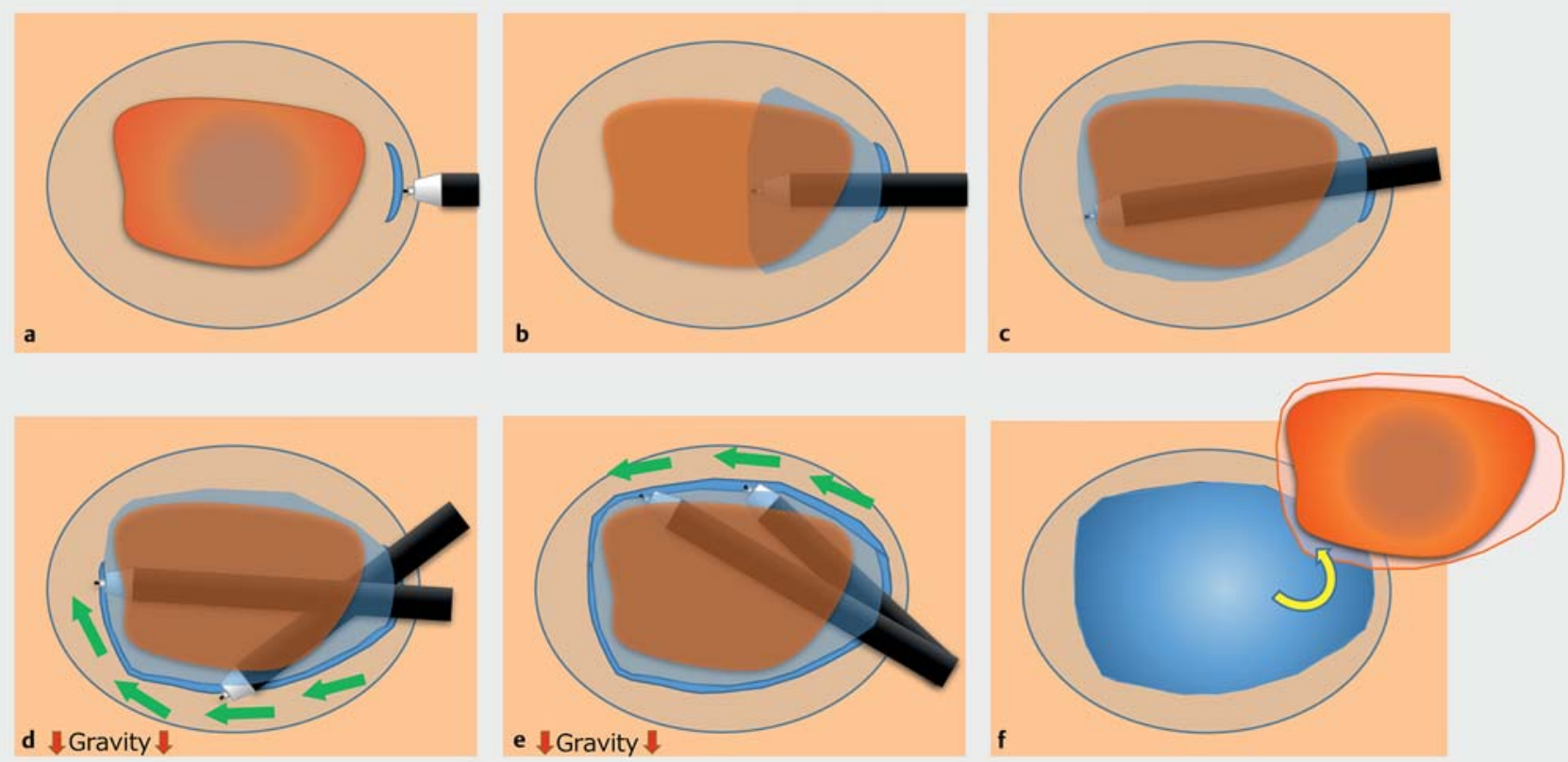

Fig. 3 Sequence for the pocket-creation method (PCM) of colorectal endoscopic submucosal dissection [13] (see also \ Video 1). a An initial mucosal incision is made approximately $20 \mathrm{~mm}$ in length approximately $10 \mathrm{~mm}$ from the distal side of the tumor after submucosal injection. b, c Creation of a submucosal pocket under most of the tumor. $\mathbf{d}$ Opening the lower side of the pocket in a step-by-step manner toward the proximal side. $\mathbf{e}$ Dissection of the remaining area in the same manner. $\mathbf{f}$ Completion of the en bloc resection.

multivariate analysis. Differences were considered statistically significant with a $P$ value $<0.05$.

\section{Results}

Seventy-three lesions were removed using the PCM and 53 lesions were removed using the CM ( $\triangleright$ Table 1$)$. All procedures were completed using the method (CM or PCM) selected at the start of the procedure. Patients in the PCM group had a significantly higher age than in the CM group (mean \pm SD, 68.9 \pm 8.2 vs. $64.8 \pm 10.8$ years, $P=0.02$ ). Use of the balloon-assisted endoscope was not significantly different when comparing the PCM and CM groups (11\% [8/73], vs. $11 \%$ [6/53]). The number

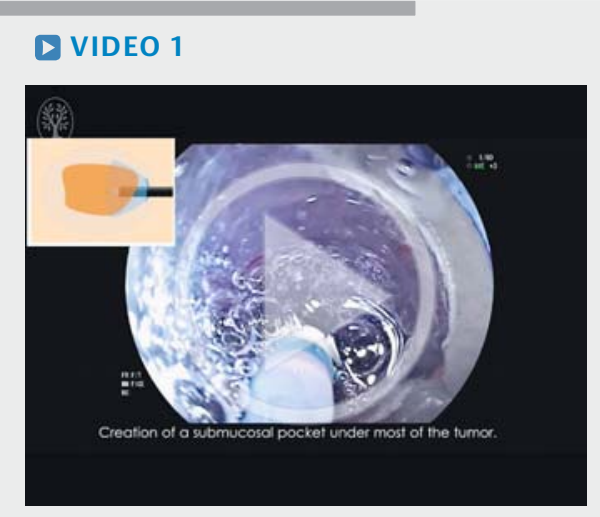

- Video 1: Sequence for the pocket-creation method (PCM) of colorectal endoscopic submucosal dissection. of procedures performed by expert endoscopists was not significantly different in the two groups (68\% [50/73], vs. $75 \%$ [40/53]) ( Table 2). Univariate analysis of preprocedure clinical characteristics of the two groups revealed that LST-NG-PD were resected more often using the PCM than the CM (45\% [33/73], vs. $26 \%$ [14/53], $P=0.04)$. Multivariate analysis identified that LST-NG-PD was significantly associated with selecting the PCM ( $\triangleright$ Table 3 ).

The en bloc resection rate in the PCM group was significantly higher than in the CM group (100\% [73/73] vs. $92 \%$ [49/53], $P=$ 0.03 ). The en bloc resection rate of the $\mathrm{CM}$ group in patients with severe fibrosis (F2) was notably lower compared to the PCM group (60\% [3/5]). The R0 resection rate was not statistically significantly different between the two groups (PCM $93 \%$ [68/73] vs. CM $91 \%$ [58/53], $P=0.74)$. Postoperative bleeding occurred after resection of one lesion in the PCM group and after two lesions in the CM group ( $1 \%[1 / 73]$ vs. $4 \%[2 / 53], P=$ 0.57). Although there were no perforations in the PCM group, two perforations occurred during the procedure in the $\mathrm{CM}$ group. Both of these were successfully treated by endoscopic clip application and antibiotics (0\% [0/73] vs. $4 \%$ [2/53], $P=$ 0.18 ). Of the lesions resected en bloc, dissection speed in the PCM group was significantly faster than in the CM group (median [IQR], $19[13-24]$ vs. $\left.14[10-22] \mathrm{mm}^{2} / \mathrm{min}, P=0.03\right)$.

\section{Discussion}

This study shows that the PCM enables high-quality and safe colorectal ESD for laterally spreading tumors, non-granular type. We achieved a $100 \%$ en bloc resection rate without per- 
- Table 1 Clinical characteristics of patients and lesions.

\begin{tabular}{|c|c|c|c|c|c|}
\hline & \multicolumn{2}{|c|}{ Pocket-creation method } & \multicolumn{2}{|c|}{ Conventional method } & $P$ value \\
\hline Number of lesions & \multicolumn{2}{|l|}{73} & \multicolumn{2}{|l|}{53} & \\
\hline Age, mean $\pm S D$, years & \multicolumn{2}{|l|}{$68.9 \pm 8.2$} & \multicolumn{2}{|l|}{$64.8 \pm 10.8$} & 0.02 \\
\hline Age $\geq 65$ years, $n(\%)$ & \multicolumn{2}{|l|}{$50(68)$} & \multicolumn{2}{|l|}{$30(57)$} & 0.19 \\
\hline Gender, male:female, n (\%) & \multicolumn{2}{|l|}{$53(73): 20(27)$} & \multicolumn{2}{|l|}{$40(75): 13(25)$} & 0.84 \\
\hline Tumor location, n (\%) & & & & & 0.83 \\
\hline Right colon & \multicolumn{2}{|l|}{$40(55)$} & \multicolumn{2}{|l|}{$32(60)$} & \\
\hline Left colon & \multicolumn{2}{|l|}{$25(34)$} & \multicolumn{2}{|l|}{$16(30)$} & \\
\hline Rectum & \multicolumn{2}{|l|}{$8(11)$} & \multicolumn{2}{|l|}{$5(9)$} & \\
\hline Macroscopic type, n (\%) & & & & & 0.04 \\
\hline Flat-elevated type & \multicolumn{2}{|l|}{$40(55)$} & \multicolumn{2}{|l|}{$39(74)$} & \\
\hline Pseudo-depressed type & \multicolumn{2}{|l|}{$33(45)$} & \multicolumn{2}{|l|}{$14(26)$} & \\
\hline Tumor diameter, median (IQR), mm & \multicolumn{2}{|l|}{$27(21-32)$} & \multicolumn{2}{|l|}{$25(20-33)$} & 0.40 \\
\hline Tumor diameter $\geq 30 \mathrm{~mm}, \mathrm{n}(\%)$ & \multicolumn{2}{|l|}{$26(36)$} & \multicolumn{2}{|l|}{$17(32)$} & 0.85 \\
\hline Expert endoscopist performed, $\mathrm{n}(\%)$ & \multicolumn{2}{|l|}{$50(68)$} & \multicolumn{2}{|l|}{$40(75)$} & 0.43 \\
\hline Prior biopsy, n (\%) & \multicolumn{2}{|l|}{$31(42)$} & \multicolumn{2}{|l|}{$16(30)$} & 0.19 \\
\hline Residual tumor, $\mathrm{n}(\%)$ & \multicolumn{2}{|l|}{$2(3)$} & \multicolumn{2}{|l|}{$1(2)$} & 1.00 \\
\hline Submucosal fibrosis, n (\%) & & & & & 0.38 \\
\hline F0 (none) & $21(29)$ & & $17(32)$ & & \\
\hline F1 (mild) & $49(67)$ & & $31(58)$ & & \\
\hline F2 (severe) & $3(4)$ & & $5(9)$ & & \\
\hline Hyaluronic acid solution injected, median (IQR), mL & $39(30-56)$ & & $34(27-58)$ & & 0.32 \\
\hline Balloon assisted endoscopy used, $n$ (\%) & $8(11)$ & & $6(11)$ & & 1.00 \\
\hline Pathological findings, n (\%) & & & & & 0.40 \\
\hline Adenoma & $20(27)$ & $58(79)$ & $25(47)$ & $38(72)$ & \\
\hline Intramucosal carcinoma & $38(52)$ & & $13(25)$ & & \\
\hline Slightly invasive $(<1000 \mu \mathrm{m})$ submucosal carcinoma & $11(15)$ & $15(21)$ & $10(19)$ & $15(28)$ & \\
\hline Deeply invasive $(\geq 1000 \mu \mathrm{m})$ submucosal carcinoma & $4(5)$ & & $5(9)$ & & \\
\hline En bloc resection, $n(\%, 95 \% \mathrm{Cl})$ & $73(100,96-100)$ & & $49(92,82-98)$ & & 0.03 \\
\hline R0 resection, $\mathrm{n}(\%, 95 \% \mathrm{Cl})$ & $68(93,85-98)$ & & $48(91,79-97)$ & & 0.74 \\
\hline Bleeding, $\mathrm{n}(\%, 95 \% \mathrm{Cl})$ & $1(1,0-7)$ & & $2(4,1-13)$ & & 0.57 \\
\hline Perforation, n (\%, $95 \% \mathrm{Cl})$ & $0(0,0-4)$ & & $2(4,1-13)$ & & 0.18 \\
\hline Dissection speed, median (IQR), $\mathrm{mm}^{2} / \mathrm{min}$ & $19(13-24)$ & & $14(10-22)$ & & 0.03 \\
\hline
\end{tabular}

forations using the PCM. ESD was accomplished more quickly using the PCM than the CM.

LST-NG are frequently accompanied by multifocal submucosal invasion, the foci of which are often difficult to predict [8]. According to the 2014 Japanese Society for Cancer of the Colon and Rectum (JSCCR) Guidelines for the Treatment of Colorectal Cancer [20], if any of the following findings are observed during histological examination of an endoscopically resected speci- men, surgical resection should be considered for additional treatment: (1) vertical tumor margin positive; (2) submucosal (SM) invasion depth $\geq 1000 \mu \mathrm{m}$; (3) vascular invasion positive; (4) poorly differentiated adenocarcinoma, signet-ring cell carcinoma, or mucinous carcinoma; (5) tumor budding grade 2 or 3 at the site of deepest invasion [21]. Therefore, en bloc resection is required in order to obtain an accurate pathological evaluation. However, if the resected submucosal layer is insufficient 
- Table2 Procedural experience of each endoscopist.

\begin{tabular}{|l|l|l|l|}
\hline $\begin{array}{l}\text { Experience with } \\
\text { colorectal ESD } \\
\text { before this study } \\
\text { period (number } \\
\text { of lesions) }\end{array}$ & $\begin{array}{l}\text { Number } \\
\text { of endos- } \\
\text { copists } \\
(\mathbf{n = 1 3 )}\end{array}$ & $\begin{array}{l}\text { Number of } \\
\text { PCM in the } \\
\text { study period } \\
(\mathbf{n}=\mathbf{7 3 )}\end{array}$ & $\begin{array}{l}\text { Number of } \\
\text { CM in the } \\
\text { study } \\
\text { period } \\
(\mathbf{n = 5 3 )}\end{array}$ \\
\hline$\geq 50$ & 6 & 50 & 40 \\
\hline $10-49$ & 3 & 19 & 11 \\
\hline$<10$ & 4 & 4 & 2 \\
\hline $\begin{array}{l}\text { ESD, endoscopic submucosal dissection; PCM, pocket-creation method; CM, } \\
\text { conventional method. }\end{array}$
\end{tabular}

or damaged by thermocoagulation in the resected specimen, it is difficult to make an exact pathological diagnosis. The PCM facilitates recognition of the muscularis and tangential approach to the muscularis, resulting in a safe and appropriate dissection line just above the muscularis [13]. This facilitates obtaining a high quality pathology specimen with a thick submucosal layer.

In this study, dissection speed in the PCM group was also significantly faster than in the CM group. One explanation for the fast dissection speed is that the PCM facilitates a tangential approach regardless of the location. Imai et al. reported that the presence of an underlying semilunar fold was one of the independent predictors of failure of en bloc resection or incidence of perforation [22]. One of the reasons for this difficulty is that the angle of the mucosal layer is dramatically changed on a semilunar fold. However, an operator using the PCM can maintain a safe tangential approach to the muscularis just like changing the direction of a jacket pocket using one's hands. Even if the muscularis is approached vertically, the vertical approach can be changed to a tangential approach by inserting the tip of the endoscope into the pocket [13]. Once the tip of the endoscope is inserted into the submucosal layer, it is spontaneously fixed. The fixed tip of the endoscope with a transparent hood easily provides traction and counter-traction, resulting in a rapid submucosal dissection with clear visualization of the muscularis [6].

Takeuchi et al. reported that poor lifting after submucosal injection is the risk factor most commonly associated with technical difficulties and adverse events during colorectal ESD [11]. Poor lifting after submucosal injection is associated with fibrosis in the submucosal layer. One of the reasons for this difficulty is the dispersion of the injected fluid after making a semi-circumferential mucosal incision when using the CM. However, when performing the PCM, injected fluid maintains elevation of the submucosal layer for a long time because the minimal mucosal incision prevents fluid dispersion. Rectal tumors extending to the dentate line are considered difficult to remove endoscopically because of the narrow lumen and the difficulty associated with a tangential approach [22]. The PCM can overcome these difficulties without a reversal operation. In that case, it is important to perform sufficient intrarectal degassing.
- Table 3 Impact of pretreatment factors on selecting the pocketcreation method (multivariate analysis).

\begin{tabular}{|l|l|l|l|}
\hline & $\begin{array}{l}\text { Odds } \\
\text { ratio }\end{array}$ & $\begin{array}{l}\text { 95\% confi- } \\
\text { dence interval }\end{array}$ & P value \\
\hline Age $\geq 65$ years & 1.65 & $(0.76-3.61)$ & 0.21 \\
\hline Female & 1.26 & $(0.53-3.01)$ & 0.61 \\
\hline Location: right colon & 0.80 & $(0.37-1.74)$ & 0.57 \\
\hline Pseudo-depressed type & 2.72 & $(1.18-6.27)$ & 0.02 \\
\hline Tumor diameter $\geq 30 \mathrm{~mm}$ & 1.43 & $(0.63-3.22)$ & 0.39 \\
\hline Expert endoscopist & 0.73 & $(0.33-1.63)$ & 0.44 \\
\hline performed & 2.14 & $(0.96-4.80)$ & 0.06 \\
\hline Prior biopsy & 1.29 & $(0.11-15.6)$ & 0.84 \\
\hline Residual tumor & & & \\
\hline
\end{tabular}

The feasibility of endoscopic submucosal tunnel dissection for esophageal tumors has been reported [23, 24]. Endoscopic submucosal tunnel dissection uses dissection in the submucosal layer in a long cylindrical manner and not a complete ESD strategy, including the manner and sequence of mucosal incision. It is useful for performing ESD in a structure with a narrow linear lumen such as the esophagus. The PCM not only includes dissection to create a wide pocket under the lesion after minimal mucosal incision, but also includes the ESD strategy with an additional mucosal incision from the lower to upper side, with gravity as a guide. Therefore, the PCM can be used in organs with complex, wide structures such as the stomach and colorectum.

We currently perform ESD for all types of colorectal lesions using the PCM, and have already reported the feasibility of using ESD for subpedunculated colorectal neoplastic lesions [12]. We have been able to carry out ESD of colorectal LST granular types safely. However, it is difficult to clarify the usefulness of the PCM for colorectal subpedunculated neoplastic lesions and LST granular types statistically. This may be partially due to the small number of colorectal subpedunculated neoplastic lesions. ESD of colorectal LST granular type lesions was easier than for LST-NG. Generally, most LST granular type lesions have no significant fibrosis.

Colorectal ESD is considered to be an advanced endoscopic technique which is difficult in the colon because of its thin wall and complex folds. In most institutions, only a few well trained endoscopists can perform colorectal ESD. In our institution, endoscopy trainees are able to perform colorectal ESD through the establishment of the PCM as a safe and uniform therapeutic strategy. In this study, ESD of colorectal LST-NG using PCM provides satisfactory outcomes even when performed by trainee endoscopists.

This study has several limitations. First, this is a retrospective study. Use of the PCM tended to be selected during the latter part of the study period. There is a possibility that performance improved with increased experience over time. Second, this is a single-center study. The results may not be generalizable because the endoscopist in this study had extensive experience 
with colorectal ESD. Less-experienced endoscopists who participated in this study had on-site assistance from highly experienced experts in performing colorectal ESD. Third, this study does not include an evaluation of long-term outcomes.

In conclusion, ESD using the PCM achieves reliably efficient resection of colorectal LST-NG. The PCM has the potential to become the standard approach for colorectal ESD. The findings in this retrospective review justify a future prospective multicenter study using the PCM for the resection of colorectal ESD.

\section{Competing interests}

H.Y. has patents for the double-balloon endoscope and ST hood described in this article. H.Y. also has a consultant relationship in FUJIFILM Corp, and has received honoraria, grants and royalties from the company. Y.M. and Y.H. have received honoraria from FUJIFILM Corp. The other authors have no disclosures relevant to this publication.

\section{References}

[1] Torre LA. Bray F. Siegel RL et al. Global cancer statistics, 2012. CA Cancer J Clin 2015; 65: 87-108

[2] Zauber AG. Winawer S]. O’Brien M] et al. Colonoscopic polypectomy and long-term prevention of colorectal-cancer deaths. NEJM 2012; 366: 687-696

[3] Woodward TA. Heckman MG. Cleveland P et al. Predictors of complete endoscopic mucosal resection of flat and depressed gastrointestinal neoplasia of the colon. Am J Gastroenterol 2012; 107: 650 654

[4] Central Social Insurance Medical Council, Ministry of Health, Labour and Welfare. The Diagnosis Procedure Combination database, a national representative medical database in Japan. 2015: Available from: http://www.mhlw.go.jp/stf/shingi2/0000104146.html (Accessed 7 May 2016)

[5] Saito Y. Fukuzawa M. Matsuda T et al. Clinical outcome of endoscopic submucosal dissection versus endoscopic mucosal resection of large colorectal tumors as determined by curative resection. Surg Endosc 2010; $24: 343-352$

[6] Shinozaki S. Hayashi Y. Lefor AK et al. What is the best therapeutic strategy for colonoscopy of colorectal neoplasia? Future perspectives from the East Dig Endosc 2016; 28: 289-295

[7] Burgess NG. Bourke M]. Endoscopic resection of colorectal lesions: The narrowing divide between East and West. Dig Endosc 2016; 28: $296-305$

[8] Uraoka T. Saito Y. Matsuda T et al. Endoscopic indications for endoscopic mucosal resection of laterally spreading tumours in the colorectum. Gut 2006; 55: $1592-1597$

[9] Tanaka S. Kashida H. Saito Y et al. JGES guidelines for colorectal endoscopic submucosal dissection/endoscopic mucosal resection. Dig Endosc 2015; 27: 417-434
[10] Toyonaga T. Man-i M. Fujita T et al. Retrospective study of technical aspects and complications of endoscopic submucosal dissection for laterally spreading tumors of the colorectum. Endoscopy 2010; 42: $714-722$

[11] Takeuchi Y. lishi H. Tanaka S et al. Factors associated with technical difficulties and adverse events of colorectal endoscopic submucosal dissection: retrospective exploratory factor analysis of a multicenter prospective cohort. Int J Colorectal Dis 2014; 29: 1275-1284

[12] Hayashi Y. Sunada K. Takahashi H et al. Pocket-creation method of endoscopic submucosal dissection to achieve en bloc resection of giant colorectal subpedunculated neoplastic lesions. Endoscopy 2014; 46: (Suppl. 01): E421-422

[13] Hayashi Y. Miura Y. Yamamoto H. Pocket-creation method for the safe, reliable, and efficient endoscopic submucosal dissection of colorectal lateral spreading tumors. Dig Endosc 2015; 27: 534- 535

[14] Hayashi Y. Shinozaki S. Sunada K et al. Efficacy and safety of endoscopic submucosal dissection for superficial colorectal tumors more than $50 \mathrm{~mm}$ in diameter. Gastrointest Endosc 2016; 83: 602 - 607

[15] Yamamoto H. Endoscopic submucosal dissection for colorectal tumors. In: Mönkemüller K. Wilcox CM. Muñoz-Navas M eds. Interventional and therapeutic gastrointestinal endoscopy. Basel: Karger; 2010: $287-295$

[16] Matsumoto A. Tanaka S. Oba S et al. Outcome of endoscopic submucosal dissection for colorectal tumors accompanied by fibrosis. Scand J Gastroenterol 2010; 45: 1329-1337

[17] Higashimaya M. Oka S. Tanaka S et al. Outcome of endoscopic submucosal dissection for gastric neoplasm in relationship to endoscopic classification of submucosal fibrosis. Gastric Cancer 2013; 16: 404 410

[18] Hayashi N. Tanaka S. Nishiyama S et al. Predictors of incomplete resection and perforation associated with endoscopic submucosal dissection for colorectal tumors. Gastrointest Endosc 2014; 79: 427 435

[19] Kanda Y. Investigation of the freely available easy-to-use software 'EZR' for medical statistics. Bone Marrow Transplant 2013; 48: 452 458

[20] Watanabe T. Itabashi M. Shimada Y et al. Japanese Society for Cancer of the Colon and Rectum (JSCCR) Guidelines 2014 for treatment of colorectal cancer. Int J Clin Oncol 2015; 20: 207-239

[21] Ueno H. Mochizuki H. Hashiguchi Y et al. Risk factors for an adverse outcome in early invasive colorectal carcinoma. Gastroenterology 2004; 127: 385-394

[22] Imai K. Hotta K. Yamaguchi Y et al. Preoperative indicators of failure of en bloc resection or perforation in colorectal endoscopic submucosal dissection: implications for lesion stratification by technical difficulties during stepwise training. Gastrointest Endosc 2016; 83: 954-962

[23] Pioche M. Mais L. Guillaud O et al. Endoscopic submucosal tunnel dissection for large esophageal neoplastic lesions. Endoscopy 2013; 45: $1032-1034$

[24] Arantes V. Albuquerque W. Freitas Dias CA et al. Standardized endoscopic submucosal tunnel dissection for management of early esophageal tumors (with video). Gastrointest Endosc 2013; 78: 946 952 